
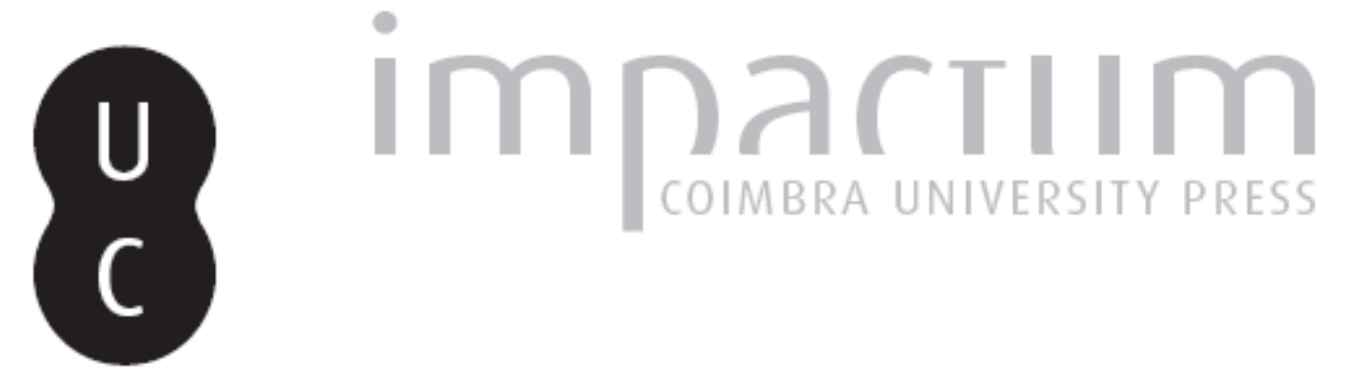

La comunità italiana a Lisbona nel regno di D. João V (1707-1750)

Autor(es): $\quad$ Alessandrini, Nunziatella

Publicado por: Imprensa da Universidade de Coimbra

URL persistente:

URI:http://hdl.handle.net/10316.2/45643

DOI: $\quad$ DOI:https://doi.org/10.14195/0870-8584_13_10

Accessed : $\quad$ 26-Apr-2023 16:34:59

A navegação consulta e descarregamento dos títulos inseridos nas Bibliotecas Digitais UC Digitalis, UC Pombalina e UC Impactum, pressupõem a aceitação plena e sem reservas dos Termos e Condições de Uso destas Bibliotecas Digitais, disponíveis em https://digitalis.uc.pt/pt-pt/termos.

Conforme exposto nos referidos Termos e Condições de Uso, o descarregamento de títulos de acesso restrito requer uma licença válida de autorização devendo o utilizador aceder ao(s) documento(s) a partir de um endereço de IP da instituição detentora da supramencionada licença.

Ao utilizador é apenas permitido o descarregamento para uso pessoal, pelo que o emprego do(s) título(s) descarregado(s) para outro fim, designadamente comercial, carece de autorização do respetivo autor ou editor da obra.

Na medida em que todas as obras da UC Digitalis se encontram protegidas pelo Código do Direito de Autor e Direitos Conexos e demais legislação aplicável, toda a cópia, parcial ou total, deste documento, nos casos em que é legalmente admitida, deverá conter ou fazer-se acompanhar por este aviso. 


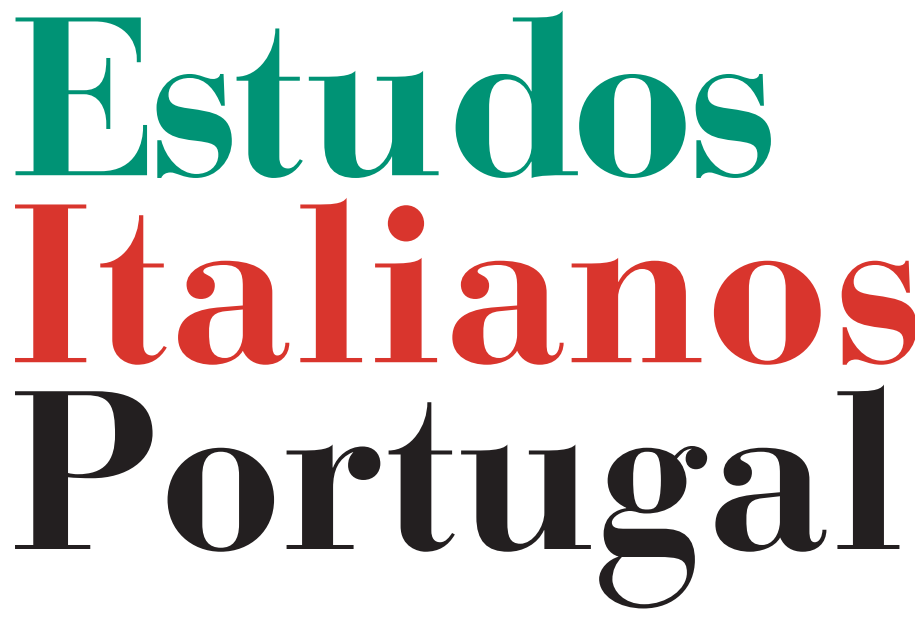

Instituto

Italiano

de Cultura

de Lisboa

Nova Série

$\mathrm{N}^{\mathbf{0}} 13$

2018 


\section{LA COMUNITÀ ITALIANA A LISBONA \\ NEL REGNO DI D. JOÃO V (1707-1750)}

NunZiatella AlesSandrini ${ }^{*}$

LO STUDIO DELLA COMUNITA italiana a Lisbona in età moderna ha visto crescere esponenzialmente, nelle ultime decadi, un interesse che già aveva dato contributi notevoli nel secolo passato. ${ }^{1}$ Tuttavia, se i secoli XV, XVI e XVII sono stati arricchiti di nuovi apporti, sia dal punto di vista di nuove fonti documentali sia dal punto di vista di nuovi approcci di ricerca, il secolo XVIII non ha meritato la stessa attenzione, soprattutto per quanto riguarda la prima metà del secolo che corrisponde, appunto, al regno di D. João V (1707-1750).

Con il presente contributo, inserito in una ricerca più ampia che sarà sviluppata attraverso un progetto presentato alla Fondazione Calouste Gulbenkian, ci si propone di abbozzare alcuni tratti della comunità italiana al tempo della corte joanina, raccogliendo dati già pubblicati e incrociando la documentazione - presente in fonti impresse $e^{2}$ e documen-

* Dottorata in Storia Moderna (Universidade Aberta de Lisboa). È ricercatrice integrata del CHAM, FCSH, Universidade Nova de Lisboa. È direttrice dell'Archivio della Chiesa di Nostra Signora di Loreto di Lisbona. lella.45@hotmail.com

${ }^{1}$ Nell'impossibilità di poter citare tutti gli apporti bibliografici che hanno avuto come oggetto lo studio della presenza italiana a Lisboa nell'età moderna, mi limito a menzionare alcuni studi che hanno implementato questo filone di ricerca presentando nuove fonti e nuove metodologie di studio. Ricordiamo, fra molti altri, Prospero Peragallo, Charles Verlinden, Virgínia Rau, Carmen M. Radulet, Marco Spallanzani, Mariagrazia Russo.

${ }^{2}$ João Luís Lisboa, Tiago C.P. dos Reis Miranda, Fernanda Olival (org.), Gazetas manuscritas da Biblioteca Pública de Évora, Lisboa, Ediçōes Colibri, vol. 1 (2002). 
tali $-^{3}$ con quella preziosa e ricca custodita nell'archivio della Chiesa di Nostra Signora di Loreto, della quale quest'anno si festeggiano i 500 anni di edificazione. Si tratta di un tema che naturalmente non può essere esaurito in poche pagine e, dunque, in questa sede si vogliono offrire spunti di riflessione presentando linee di ricerca per un tema che, per le notevoli potenzialità che presenta, merita di essere approfondito.

Pur mancando uno studio sistematizzato sulla comunità italiana a Lisbona nel periodo preso in esame in questo testo, non si può negare che la presenza di italiani alla corte joanina sia un argomento sconosciuto. Dobbiamo, infatti, tenere in conto i numerosi contributi nell'ambito dell'arte, della musica, della diffusione culturale, della diplomazia, dai quali abbiamo la nozione di quanto numerosi fossero gli "italiani" che arrivarono nella capitale portoghese, per i più disparati motivi, durante il regno di D. João $\mathrm{V}^{4}{ }^{4} \mathrm{E}$ vero, infatti, che

${ }^{3}$ Citiamo a questo proposito la raccolta di documenti provenienti dalla Câmara Eclesiástica de Lisboa (Sumários Matrimoniais), custodito in parte nell'Archivio della Torre do Tombo di Lisbona e in parte nell'Arquivo Histórico do Patriarcado de Lisboa effettuata da Rui Mendes, "Câmara Eclesiástica de Lisboa: contributos para o estudo da presença dos italianos e famílias de origem italiana na região de Lisboa (séculos XVI, XVII e XVIII)", Alessandrini, Nunziatella; Mateus, Susana Bastos; Russo, Mariagrazia, Sabatini, Gaetano (org.) "Scrigni della Memoria": Arquivos e Fundos Documentais para o estudo das Relaçôes Luso-Italianas, Lisboa, Cátedra de Estudos Sefarditas "Alberto Benveniste", 2016, pp. 115-150 e da Gonçalo Nemésio, "Os Sumários Matrimoniais da Câmara Eclesiástica de Lisboa (C.E.L.) e o Registo Geral de Testamentos (R.G.T.) (I.A.N./T.T.). Fontes para o estudo dos italianos que passaram a Portugal", id., pp. 187-222.

Un'altra importante ricerca è stata portata avanti da Pedro Pinto che ha riunito la documentazione dei fondi manoscritti della Biblioteca Nacional de Portugal riguardante la presenza italiana in Portogallo: "A "Italia" e os italianos nos fundos manuscritos da Biblioteca Nacional de Portugal: uma aproximação", in id., pp. 223-265.

${ }^{4}$ La vasta letteratura su pittori, architetti, scultori, musicisti a Lisbona nel secolo XVIII non ci permette di esporre in questa sede una bibliografia esauriente. Ricordiamo, fra molti altri, gli studi degli storici dell'arte fra cui Teresa Leonor Vale, Vítor Serrão, Giuseppina Raggi, Sandra Costa Saldanha e gli studi di musicologi, fra cui Gerhard Doderer, José Maria Pedrosa Cardoso, Cristina Fernandes, João Pedro d'Alvarenga. 
nell'ambito degli studi artistici, inglobando questi la pittura, la scultura, la musica, l'architettura, si può contare, come vedremo, su di una sostenuta quantità e qualità di contributi. Nell'ambito di un approccio socio economico, siamo, al contrario, di fronte ad una ricerca che ha prodotto esigui risultati, mancando ancora solidi contributi. Si ha, così, la consapevolezza della presenza di illustri artisti italiani a Lisbona, conseguenza della politica artistica del lungo regno di D. João V, ma non si ha una visione d'insieme di questa comunità di stranieri che, come vedremo, si raggruppava principalmente nelle zone del Chiado, Encarnação, Mártires, Corpo Santo.

Raccontare la comunità italiana presente a Lisbona nella prima metà del Settecento, significa sfogliare con attenzione soprattutto i volumi dei Róis dos Confessados, (1724-1883) e gli Atti delle Riunioni della Giunta della Confraternita di Loreto. Una breve descrizione di queste due fonti indicherà la loro importanza per il fine che ci proponiamo. Nei Róis dos Confessados, il parroco annotava ogni anno il nome, provenienza, stato civile e abitazione di tutti coloro che venivano a disobbligarsi dal precetto pasquale. Per quanto riguarda i volumi degli atti delle riunioni della Giunta, questi, oltre a informare sulle attività della Confraternita di Loreto, ci presentano le persone, ovvero, i "funzionari" che reggevano le sorti della Chiesa di Loreto: un provveditore, un maggiordomo, uno scrivano, un tesoriere. ${ }^{5}$ L'elezione di tali funzionari era nelle mani di dodici votanti, scelti, così come determinato dagli Statuti, fra gli uomini più facoltosi della comunità italiana. Un altro prezioso volume, riapparso molto di

${ }^{5}$ Fino all' 8 settembre 1733, l'incarico di tesoriere era nelle mani dello scrivano; a partire da questa data passa in quelle del maggiordomo. Ringrazio padre Sergio Filippi per la precisazione e rimando al suo testo per la storia della Chiesa di Loreto di Lisbona: La Chiesa degli Italiani. Cinque secoli di presenza italiana a Lisbona negli archivi della Chiesa di Nostra Signora di Loreto, Lisboa, Fabrica da Igreja Italiana da Nossa Senhora do Loreto, 2013 
recente nell'archivio della chiesa di Loreto, precisamente il 7 maggio 2018, rappresenta il tassello mancante nella ricostruzione della comunità italiana della prima metà del Settecento a Lisbona. Si tratta del $1^{\circ}$ volume del registro dei battesimi dall'anno 1689 fino al 1748. Il volume riempe, in parte, la lacuna delle prime due decadi del Settecento ${ }^{6}$, facilitando la ricostruzione delle reti familiari e aiutando a disegnare i percorsi di relazioni sociali. Fino al suo ritrovamento, infatti, per conoscere nella maniera più precisa possibile la composizione della comunità italiana a Lisbona della prima metà del Settecento ci si doveva supportare di altra documentazione avulsa presente nell'archivio della Chiesa di Loreto o in altri archivi portoghesi e stranieri. Il volume ritrovato, o meglio, riconsegnato alla chiesa, è da questo punto di vista, e non solo, estremamente prezioso.

La lettura della documentazione ci porta a credere che se i secoli XV, XVI e XVII furono caratterizzati dall'arrivo di mercanti e uomini d'affari che stabilirono nella capitale portoghese le loro case commerciali - ricordiamo, come esempio, Marchionni, Sernigi, Affaitati (secolo XV), Giraldi, de' Bardi (secolo XVI), Ghersi, Micone, Carrega, (secolo XVII) - il secolo XVIII, e ci riferiamo in particolare al periodo joanino, si presenta "importatore" di figure non necessariamente legate al commercio e alla finanza. È bene, comunque, sottolineare che la venuta di artisti a Lisbona sempre fu un marchio distintivo della comunità italiana e, per quello che riguarda il secolo XVII, possiamo individuare due momenti che incentivarono la loro venuta nella capitale portoghese. Il primo momento deve essere senz'altro identificato con l'incendio del 1651 che devastò la chiesa di Loreto. La confraternita si mise subito in movimento per ripristinarne la bellezza originaria, promuovendo l'arrivo di artisti italiani e ordinan-

\footnotetext{
${ }^{6}$ Ricordiamo, infatti, che i volumi dei Róis dos Confessados iniziano a partire dall'anno 1724 .
} 
do materiali e opere varie dall'Italia. ${ }^{7}$ Un secondo momento può essere individuato a cavallo fra la fine del secolo XVII e inizio del seguente, quando il clima di una raggiunta stabilità e di un certo arricchimento aveva sostituito gli anni tragici delle guerre di restaurazione.

Tornando alla comunità italiana che viveva a Lisbona nella prima metà del Settecento, possiamo affermare che questa era costituita, in parte, dai discendenti delle famiglie che già da tempo popolavano la capitale lusitana. Ricordiamo le famiglie di commercianti degli Spinola, Centurione, de' Ferrari, Lercaro, Conti Ventimiglia, Micone, Carrega, Ghersi, Ravara, Ginori, Viganego, ${ }^{8}$ fra molte altre. Nel corso del Settecento, come ben sottolinea Francesca Trivellato, "il porto lusitano si affermò come il principale centro di scambio tra Livorno e la penisola iberica" e i commercianti italiani a Lisbona approfittarono questo intercambio, costituendo società commerciali con il ruolo di intermediari fra Lisbona e il porto italiano. Tra queste società, ricordiamo, fra altre, quella composta da Paolo Girolamo de' Medici ${ }^{10}$ e Luigi Niccolini,

${ }^{7}$ Pensiamo, per esempio, all'arrivo di Giovanni Battista Garvo, intorno al 1670, con la moglie e il figlio Carlos Baptista Garvo. Sui Garvo, cf. Teresa Leonor Vale, "Os Garvo - Uma família de artistas italianos em Lisboa e o seu papel no contexto da arte portuguesa de Seiscentos e Setecentos", N. Alessandrini, P. Flor, M. Russo, G. Sabatini (org.), Le Nove son tanto e tante buone, che dir non se ne pò. Lisboa dos Italianos: História e Arte (sécs. XIV-XVIII), Lisboa, Cátedra de Estudos Sefarditas Alberto Benveniste, 2013, pp. 175-187.

${ }^{8}$ Pietro Francesco Viganego era stato insignito dell'incarico di console dei genovesi a Lisbona nel 1692 e lo mantenne fino alla sua morte avvenuta nel 1723. Il figlio Fernando Aniceto Viganego, gli succederà nell'incarico fino al 1756.

${ }^{9}$ Francesca Trivellato, Il commercio interculturale. La diáspora sefardita, Livorno e $i$ traffici globali in età moderna, Roma, Viella, 2016, p. 285. Traduzione italiana di The Familiarity of Strangers - The Sephardic Diaspora, Livorno, and cross-cultural Trade in the early modern period, Yale University, 2009.

${ }^{10}$ Paolo Girolamo de' Medici scrisse, nel 1735, quando era provveditore della Chiesa di Loreto, una Relação da Fabrica da Igreja de Nossa Senhora do Loreto para nela se depozitar o santíssimo sacramento nas Endoenças deste presente anno de 1735 mandada fazer pelo Sr. Paulo Hieronymo de Medicis Provedor da mesma Igreja. Coimbra M.DCC.XXX. La relazione è inclusa nel Livro de Cazamentos custodito nell'archivio della Chiesa di Loreto. 
entrambi fiorentini; la società genovese Barducci, Giudici, Perini; i ricchi mercanti Eneas Beroardi, fiorentino, e Lazaro Maria Cambiaso, genovese.

Si possono individuare alcune date significative che determinarono, così ci sembra, l'intensificarsi dell'arrivo di italiani a Lisbona nelle prime decadi del Settecento. Cominciamo seguendo la riflessione della storica dell'arte Giuseppina Rag$\mathrm{gi}^{11}$ che propone una lettura critica circa la concezione comune che identifica la figura di D. João V come foriera dell'arrivo in massa di artisti italiani a Lisbona. Non vi sono dubbi sul fascino che la cultura italiana esercitava su D. João $\mathrm{V}$ e conosciuta è la passione del monarca portoghese per la musica sacra italiana. Tuttavia, se si sposta l'attenzione sull'attività culturale promossa dalla regina Maria Anna d'Asburgo (1708-1754), ${ }^{12}$ si può facilmente riconoscere la sua influenza in quanto promotrice del teatro d'opera italiano, le cui rappresentazioni erano frequenti ed usuali nella corte di Vienna, dove "La scelta dei generi musicali e la ricchezza degli allestimenti riflettevano il ruolo del festeggiato nella gerarchia sociale della corte" ${ }^{13}$ Consapevole della passione della regina, la corte portoghese organizzò, in occasione del suo arrivo a Lisbona, nell'ottobre del 1708, la rappresentazione di un'opera nel collegio gesuita di Santo Antão. In pochi anni l'opera in musica divenne consuetudine nelle principali occasioni celebrative, affiancandosi, così, alle rappresentazioni di musica sacra e richiamando dall'Italia cantanti e musicisti.

${ }^{11}$ Giuseppina Raggi, "L’effervescenza culturale del regno di Giovanni V di Portogallo (1707-1728): una visione critica controcorrente”, José Martínez Millán, Félix Labrador Arroyo, Filipa M. Valido-Viegas de Paula- Soares (org.), Decadencia o Reconfiguración? Las Monarquias de España y Portugal en el cambio de siglo (1640-1724), Madrid, Ediciones Polifemo, 2017, pp. 317-337.

12 Sulla regina-consorte Anna Maria d'Asburgo, cf. Susana Munch Miranda e Tiago C.P. dos Reis Miranda, A rainha arquiduquesa. Maria Ana de Austria, Maia, Círculo de Leitores, 2014.

${ }^{13}$ Giuseppina Raggi, "L'effervescenza culturale del regno di Giovanni V di Portogallo (1707-1728): una visione critica controcorrente”, cit., p. 318. 
Un altro anno significativo in quanto input dell'arrivo di italiani a Lisbona fu il 1716 quando la cappella reale venne elevata a patriarcale, producendo la divisione della diocesi di Lisbona in orientale e occidentale, quest'ultima situata tra il Terreiro do Paço e Corpo Santo. L'11 dicembre 1716, il vescovo di Porto D. Tomás de Almeida ricevette l'avviso del Segretario di Stato della nomina, da parte di Sua Maestà, di Patriarca e Arcivescovo della Cappella Reale elevata a Patriarcale da Sua Santità. ${ }^{14}$ Il 16 dicembre 1716, il Dottor João Cardoso Castelo ricevette la nomina di Vicario generale della nuova Diocesi Patriarcale di Lisbona Occidentale, e il 24 dicembre dello stesso anno venne cantato il Te Deum per la grazia ricevuta. ${ }^{15}$ Non possiamo soffermarci in questa sede sul significato che l'elevazione della cappella Reale a Patriarcale produsse sulla nuova connotazione della capitale portoghese, ma è certo che costituì un importante momento che portò a Lisbona, fra altri, artisti del calibro dell'architetto siciliano Filippo Juvarra e del compositore toscano Domenico Scarlatti. ${ }^{16}$ Dell'arrivo di musicisti italiani a Lisbona si parla nella corrispondenza mantenuta dall'allora Nunzio a Lisbona, Monsignor Bichi che rimase a Lisbona dal $1710 \mathrm{al}$ 1720. In una missiva del 12 settembre 1719, il Nunzio riporta che il monarca aveva fatto preparare " di tutto punto, con la sua solita Real generosità, una gran casa vicino alla Chiesa di Loreto per i Musici, che à momento si attendono qui di Roma, tanto nazionali che della Cappella Pontifícia, et altri, nella quale saranno per un mese trattati à spese Regie, doppo del quale, dovroanno pensare á mantenersi con il soldo

${ }^{14}$ Gazeta de Lisboa, 12-12-1716

${ }^{15}$ Gazeta de Lisboa, 26-12-1716

${ }^{16}$ Cf. Giuseppina Raggi, "Lasciare l’Orma: os passos de Filippo Juvarra na cidade de Lisboa", in Nunziatella Alessandrini et alii, Le Nove son tanto e tante buone, che dir non se ne pò, cit., p. 192. Raggi sottolinea l'importanza delle relazioni fra i due artisti in Italia considerando non trattarsi di una coincidenza il loro arrivo alla corte joanina nel 1719. Cf., pp. 196 e seg. 
stabilitoli” ${ }^{17}$ Nella stessa missiva, il Nunzio riferisce che "con grand.mo concorso di Popolo, fu celebrato da Mons.r Nunzio solenne Pontificale nella Chiesa di Na Sig.ra di Loreto della Nazione Italiana li 8. del Corrente, in cui si festeggiaua la Natiuità della Beatíssima Vergine, e la detta Nazione oltre alla magnificenza dell'apparato fece riuscire più sontuosa la Funzione con una strepitosa musica tutta sull'uso italiano". ${ }^{18}$ Il 14 settembre giunsero "per la via del mare, i Musici tanto desiderati da questa Corte, e subito si feccio intendere, che hauevano promessa à San Filippo Neri una Missa solenne in musica per il loro felice arriuo dentro la Barra". ${ }^{19}$ La messa nella Patriarcale fu talmente applaudita che la generosità del monarca si manifestò ancora una volta con la donazione di 1000 scudi romani da dividersi fra i nove cantanti italiani. Fra i cantanti, la presenza di castrati aveva risvegliato un'enorme curiosità fra la gente che " i primi giorni correua alla loro casa (...) per uederli come per merauiglia”. ${ }^{20} \mathrm{Nel}$ la missiva del 10 ottobre 1719 , il Nunzio riferisce che il re stava aspettando con ansia l'arrivo del maestro di Cappella Domenico Scarlatti e i musicisti Mossi e Floriano (si trattava sicuramente di Floriano Flori).

Fra il 1719 e il 1720 vennero assunti come cantanti della Cappella Patriarcale Antonio Natali, Girolamo Bezzi, ${ }^{21} \mathrm{Ga}-$ etano Mossi, Anzano Bernini, Giuseppe Coccucioni, Carlo

${ }^{17}$ Un importante contributo di Gerhard Doderer e Cremilde Rosado Fernandes, "A música da sociedade joanina nos relatórios da Nunciatura Apostólica em Lisboa (1706-1750)", Revista Portuguesa de Musicologia, 3, 1993, raccoglie stralci della corrispondeza dei Nunzi a Lisbona nel periodo del regno joanino relazionati con l'ambito musicale. Il fondo si trova nella sezione Portogallo della Segreteria di Stato dell'Archivio Segreto Vaticano

${ }^{18} \mathrm{Ib}$., p. 91

${ }^{19}$ Lettera del Nunzio del 26 settembre 1719.

${ }^{20} \mathrm{Ib}$.

${ }^{21} \mathrm{Nel}$ registro degli Obiti dell'archivio della Chiesa di Loreto troviamo che Jeronimo Bessi, romano, muore il 26 maggio 1735 all'età di 53 anni, ed era figlio di Sebastiano Bessi e di Caterina Fabiani. 
Cristini, Floriano Flori, Carlo Gianetti, Luigi Biancardi, Antonio Pasere, Felice Merlari. ${ }^{22}$

Scorrendo i Róis dos Confessados incontriamo altri musicisti, fra cui il bolognese Alessandro Paghetti che abitava nel palazzo Corte Real dell'Infante D. Francisco (Mártires) con il figlio Giovanni Battista ${ }^{23}$; Paolo Antonio Veraci, fiorentino, che viveva in casa di Estêvão (Stefano) Olivieri nella rua das Flores ${ }^{24}$; il napoletano Antonio Pecorari che abitava nella Cordoaria Velha e che sarebbe divenuto suocero di Alessandro Giusti; ${ }^{25}$ il milanese Giovanni Battista Clerici che abitava a S. Nicolau, ${ }^{26}$ probabilmente membro della famiglia dello scenografo Roberto Clerici che, nel 1736, mise in scena Alexandre na India di Metastasio nella Sala dell'Academia nella Piazza da Trindade.

In un altro importante documento troviamo i nomi di musicisti italiani attivi a Lisbona nella prima metà del Settecento. Si tratta del Compromisso da Irmandade da Gloriosa Virgem e Mártir Santa Cecilia (1749) ${ }^{27}$ che presenta le firme dei musicisti della confraternita di Santa Cecilia, fra i quali un gruppo di italiani, alcuni dei quali già nominati. Riporto, qui di seguito, parte dei nomi che, tuttavia, non esauriscono la lista, visto che il manoscritto presenta, oltre ad una grossa macchia nera che lo rende illeggibile, altre difficoltà di lettura: Gio Batta Mazzetti, Raffaele Magino, João Baptista e Francesco Maria Paghetti, ${ }^{28}$ João Baptista Biancardi, Mi-

${ }^{22}$ Cristina Fernandes, "Il dotto e rispettabile D. Giovanni Giorgi", illustre maestro e compositore nel panorama musicale portoghese del Settecento", in Rivista Italiana di Musicologia, vol XLVII (2012), p. 165.

${ }^{23}$ ANSL, Livro da desobrigação 1724- 1738, fl. 13v. (1726)

${ }^{24}$ ANSL, Livro da desobrigação 1724- 1738, fl. 26v. (1728)

${ }^{25}$ Alessandro Giusti sposerà, nel 1750, Teresa Maria, figlia di Antonio Pecorario. Padrino del matrimonio Luca Giovine. ANSL, Livro da desobrigação 1724- 1738, fl. 45v. (1730). Sulla presenza a Lisbona di Antonio Pecorari, Cf. Sandra Costa Saldanha, Alessandro Giusti (1715-1799) e a aula de escultura de Mafra, Tesi di dottorato Faculdade de Letras da Universidade de Coimbra, 2012, pp. 70 e seg.

${ }^{26}$ ANSL, Livro da desobrigação 1724- 1738, fl. 13 (1726)

${ }^{27}$ Biblioteca Nacional de Portugal, http://purl.pt/24981

${ }^{28}$ João Baptista e Francesco Maria Paghetti erano figli di Alessandro Paghetti e di sua moglie Camilla, originari di Bologna. 
chel Angelo Gallini, Ambrogio Grassi, Antonio Mignucci, Pedro Jorge Avondano, Serafino Atanasio Nicola Seri, Luca Giovine, ${ }^{29}$ Antonio Rapizzi, Antonio Pecorario, João Baptista Romano, Carlo Gianetti, Vincenzo Michelotti, Francesco Terci (sic), Luca Francesco Lombardi, Nicolao Palmari, José Schiavori, Tommaso Guarducci, ${ }^{30}$ Domenico Scambietti, Sebastiano Barzzi, Antonio Tedeschi.

Se l'elevazione della cappella reale a patriarcale aveva dato avvio all'arrivo di musicisti, cantanti e compositori italiani, un altro avvenimento aveva contribuito all'afflusso di italiani a Lisbona. Il 17 novembre del 1717 iniziarono i lavori per la costruzione della Basilica di Mafra, un'impresa che sarebbe durata almeno fino alla consacrazione avvenuta il 22 ottobre 1730, giorno del compleanno di D. João V, "com toda a Magnificencia Imaginável”. ${ }^{31}$ L'arrivo dall'Italia di architetti, pittori, scultori e operai qualificati ${ }^{32}$ fu determinante. Oltre alla già citata famiglia dei Garvo, menzioniamo lo scultore romano Gaetano Pace il quale abitava "na vila de Mafra trabalha

${ }^{29}$ Luca Giovine arriva a Lisbona nel 1725 . Nello stesso anno giunge a Lisbona Giovanni Giorgio (João Jorge), compositore veneziano che rimarrà a Lisbona dal $1725 \mathrm{al} 1755$ (dopo il terremoto ritornerà in patria). Cf. Cristina Fernandes, "Il dotto e rispettabile D. Giovanni Giorgi”, cit., pp. 157-203.

${ }^{30}$ Tommaso Guarducci fu padrino di battesimo di Maria Vittoria, figlia di Antonio Pecorari battezzata a Loreto nel 1750 Cf. Sandra Costa Saldanha, cit., p. 77.

${ }^{31}$ Lettera del 25 ottobre del 1730 inviata da Lisbona da José Correia de Abreu a Fr. José Maria da Fonseca Évora a Roma. Biblioteca Nacional de Portugal (BNP), Mss 41, n.7 Secção de Reservados. Nel manoscritto sono raccolte le lettere inviate da Lisbona da José Correia de Abreu a Fr. José Maria da Fonseca Évora a Roma da dicembre 1728 fino a novembre 1734. Stralci delle lettere sono pubblicate da A. Ayres de Carvalho, D. João Ve a arte do seu tempo, edição do autor, 1962, vol. II, p. 412.

${ }^{32}$ A questo proposito, voglio ricordare un membro della famiglia genovese dei Massa, molto numerosa a Lisbona. Domenico Massa era probabilmente un maestro falegname e fu contattato da D. João $\mathrm{V}$ per preparare il sostegno in legno delle campane della Basilica di Mafra. Cf. Nunziatella Alessandrini, "Revelando Domenico Massa. Contributo para a História da Vivência de um Genovês no Estaleiro da Real Obra de Mafra e Devoto da Ordem Terceira da Penitência", in Mafra Sacra-Memória e Património, Mafra, Zéfiro, 2017, pp. 326-335. Il volume è stato redatto in occasione delle commemorazioni dei 300 anni della costruzione della Basilica di Mafra. 
nas obras de S. Mag." ${ }^{33}$ Nel 1733, lo troviamo che abitava a Lisbona, alla Cotovia, (Mercês). ${ }^{34}$ Ancora, lo scultore originario di Massa Carrara Alessandro Tanzi, e, nella seconda metà degli anni Quaranta il già citato Alessandro Giusti. ${ }^{35}$

Oltre alla notevole presenza di artisti italiani a Lisbona, l'apprezzamento di D. João V per la cultura straniera si può confermare anche attraverso l'emanazione del nuovo Regimento che, dal 1572, governava l'attività dei librai a Lisbona. La proposta del nuovo Regimento del 1733 presentata al senato del Comune di Lisboa non alterava in modo sostanziale il Regimento antico, ma facilitava l'attività agli stranieri, ${ }^{36} \mathrm{i}$ quali mantenevano una posizione sempre più di rilievo dovuta alla politica culturale joanina.

Dal testamento del torinese João José Guibert, libraio, la cui apertura avviene il 23 maggio 1736, possiamo estrapolare nomi di piemontesi residenti nella capitale portoghese: João Jacomo Nora residente nella Cordoaria Velha (Mártires), João Baptista Piano, Jacomo Martins, Andrea Conte, Michel Antonio Michelotti e João Batista Guinioens, tutti abitanti nella medesima casa nella Cordoaria Velha. Era qui che viveva anche Guibert e nello stesso quartiere aveva un negozio, "Guibert e Reysand" la cui pubblicità era promossa nella Gazeta de Lisboa de $1732 .{ }^{37}$ Sappiamo inoltre che João José Guibert, sepolto nella chiesa di Nostra Signora di Loreto di Lisbona, era fratello di Maria Lucrezia Merlo sposata con Jacome Merlo, genovese.

${ }^{33}$ ANSL, Livro da desobrigação 1724- 1738, fl. 28v.

${ }^{34}$ ANSL, Livro da desobrigação 1724- 1738, fl. 70v.

${ }^{35}$ Cf. Sandra Costa Saldanha, "Alessandro Tanzi: um escultor de Carrara na Lisboa de Setecentos" in Revista de História da Arte, n. 11 (2014) pp. 352-369; Id., Alessandro Giusti( 1715-1799) e a aula de escultura de Mafra, cit.

${ }^{36} \mathrm{La}$ trascrizione dei Regimentos dei librai del 1572 e 1733 si trova in Fernando Guedes, Os livreiros em Portugal e as suas associações desde o século XV até aos nossos dias, Lisboa, Verbo, 1993, pp. 125-129 e 134-144.

${ }^{37}$ Manuela D. Domingos, Livreiros de Setecentos, Lisboa, Biblioteca Nacional, 2000, p. 24. 
Altri genovesi nell'ambito della diffusione culturale: i Lerzo e i Gnecco, librai e impressori, gli Ottone e Gambino cartai. Uomini d'affari, artisti, artigiani, non si esaurisce certo con questi nomi la variegata ed effervescente comunità italiana che, prima del devastante terremoto del 1755 , popolava le vie della capitale portoghese e della quale si è voluta fare una breve rassegna. 